\title{
Delegating authority to officials of the institution
}

W hile John Callahan is technically an "official of the institution," whether he has been delegated authority to revoke an IACUC approval is an entirely different matter. Though PHS Policy states that protocols "may be subject to further appropriate review and approval by officials of the institution, ${ }^{1,}$ such officials are not automatically given the authority to administratively suspend animal activities - that authority must be delegated to them by the Institutional Official (IO).

The regulations are silent on how institutions can develop their own mechanisms for further institutional review of animal use activities. Ideally, the Great Eastern University IACUC would have a policy that specifies which university officials-besides the IACUC and IO-have the authority to halt, stop, or (administratively) suspend an animal use activity. If such a policy exists, the IACUC should refer Callahan to the procedures described in the policy and follow it accordingly.

If Callahan has been delegated this authority, the IACUC should clarify whether he intends to revoke approval of the entire protocol or to just stop the procedures that will eventually be replaced with the simulation lab. Regardless, the IACUC should inquire as to whether his concerns were discussed with the medical school administration and protocol PI. The IACUC should then engage the IO, Callahan, medical school, and protocol PI in a discussion of Callahan's concerns, which should have been addressed by the IACUC during review of the protocol.

As the Chair stated, the IACUC had considered whether the final teaching demonstrations were necessary, knowing that a new simulation lab would soon replace the use of animals for those demonstrations. The IACUC would have required documentation in the protocol for consideration of the 3Rs (Replacement, Reduction, and Refinement, as described by Russell and Burch ${ }^{2}$ ) regarding painful and distressful procedures involving USDA-covered species ${ }^{3}$. For Replacement, it would have been appropriate to consider the cardiothoracic simulation laboratory as an alternative method.

The IACUC, IO, PI, and medical school administration should discuss with Callahan the timing of the teaching curriculum relative to the opening of the new simulation laboratory. If these do not align and the order of teaching demonstrations are critical to student learning outcomes, it would make sense to allow a final set of animals to be used. The Great Eastern IACUC could view this as an opportunity to show how the Committee considers and engages university colleagues on the ethical and humane use of animals in Great Eastern University's teaching activities.

Melodie Blakemore ${ }^{凶}$ and Sean Mayuga University of California Santa Barbara, Santa Barbara, CA, USA.

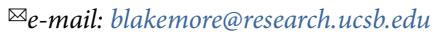

Published online: 24 April 2020

https://doi.org/10.1038/s41684-020-0532-2

References

1. Public Health Service. PHS Policy on Humane Care and Use of Laboratory Animals. (U.S. Department of Health and Human Services, National Institutes of Health, Bethesda, MD, 2015).

2. Russell, W. M. S. \& Burch, R. L. The Principles of Humane Experimental Technique. (Methuen, London, 1959).

3. Animal Welfare Act. Public Law 89-544, 1966, as amended (P.L. 91-579, P.L. 94-279, and P.L. 99-198), 7 U.S.C. 2131 et seq. Implementing regulations are published in the Code of Federal Regulations (CFR), Title 9, Chapter 1, Subchapter A, Parts 1, 2, and 3. 